- In Deutschland lagen diese Werte bei 59 und 46 Prozent.

Die breite Mehrheit der Internet-Nutzer (84 Prozent) möchte nicht, dass jemand über ihre Gewohnheiten Bescheid weiß, solange sie selbst diese Informationen nicht wissentlich freigeben.

Verbraucher erwarten künftig weniger Datenschutz Das Vertrauen der Menschen in den Schutz ihrer Daten sinkt. Dies belegen die folgenden Ergebnisse:

Weltweit stimmten 59 Prozent der Teilnehmer der Frage zu, ob sich ihr Datenschutz im Vergleich zum Vorjahr verschlechtert habe.

- In Brasilien und den USA hatten 71 bzw. 70 Prozent der Teilnehmer das Gefühl, dass sie jetzt weniger Datenschutz genießen.

- In Deutschland hatten 50 Prozent der Teilnehmer dieses Gefühl.

- Frankreich war das einzige Land, in dem weniger als die Hälfte der Befragten angab, weniger Datenschutz zu genießen. 42 Prozent hatten diese Wahrnehmung.

Eine große Mehrheit von 81 Prozent der Befragten erwartet, dass der Datenschutz in den nächsten fünf Jahren weiter abnehmen wird. In Deutschland betrug dieser Wert sogar 88 Prozent.

\section{Kaspersky Lab: Sicherheitsregeln beim Download, der Verwaltung und Nutzung von Apps}

Über App-Stores kommen Smartphone- und Tablet-Anwender in den Genuss zahlreicher Spiele und Werkzeuge. Allerdings sind auch gefährliche Apps und Schadprogramme im Umlauf. Kaspersky Lab zeigt, auf was mobile Nutzer bezüglich der IT-Sicherheit beim Download und der Nutzung von Apps achten sollten.

Kaspersky Lab unterscheidet zwischen rein mobilen Schadprogrammen und schädlichen Apps. Cyberkriminelle nutzen schädliche Apps vor allem, um Schadprogramme zu verbreiten. Zudem verfügt die Mehrheit der schädlichen Apps über Botnetzfunktionalitäten. Damit können sie zeitnah auf neue Anforderungen reagieren und neue Funktionen für die Schadsoftware hinzufügen. Bei Google Play werden derzeit über 1.200.000 Apps angeboten. In inoffiziellen App-Stores finden sich deutlich mehr Apps zum Download - allerdings auch mehr schädliche. Kaspersky Lab hat mehr als zehn Millionen dubiose Apps identifiziert, mit denen Cyberkriminelle beispielsweise Schadcode verbreiten.

Kaspersky Lab kennt aktuell 347.525 einzigartige mobile Schädlinge und 841 Schädlingsfamilien. Davon haben es 99 Prozent auf Android abgesehen. Zeitgleich stellt Kaspersky Lab einen Anstieg in punkto Qualität und Lokalität der Schadprogramme fest. Vor allem mobile Banking-Trojaner sind auf dem Vormarsch. Ende des vergangenen Jahres kannte Kaspersky Lab noch 1.321 einzelne mobile Banking-Trojaner. Ende des ersten Quartals 2014 waren es bereits 2.503 solcher Schädlinge

Mit den folgenden Sicherheitsregeln schützen sich Smartphoneund Tablet-Nutzer vor schädlichen Apps und Schadprogrammen sowie vor unerwünschtem Abfluss persönlicher Informationen.

Erste Sicherheitsregel: die Reputation einer App prüfen Smartphone- und Tablet-Anwender sollten grundsätzlich die Vertrauenswürdigkeit einer App prüfen - beispielsweise über viele gute Bewertungen. Nutzer sollten brandneuen Apps mit negativen Bewertungen aus dem Weg gehen. Den Erstdownload einer App sollten Nutzer vermeiden.
Zweite Sicherheitsregel: App-Store-Einkäufe über Passwörter oder Biometrie absichern

Sowohl Apple als auch Android-Nutzer sollten für alle App-StoreEinkäufe die Passwortschutzfunktionalität oder einen biometrischen Schutz festlegen - entweder nativ oder über eine App.

Dritte Sicherheitsregel: In-App-Einkäufe kontrollieren Vor allem kostenlose Apps operieren oft mit zusätzlichen Einkäufen innerhalb der App - beispielweise wenn man bei mobilen Spielen für den Zugang zu einem neuen Level oder eine neue Waffe bezahlt. Android- und iOS-Anwender können unbeabsichtigte InApp-Einkäufe - beispielsweise ihrer Kinder - über die Geräteeinstellungen vermeiden.

Vierte Sicherheitsregel: Apps ausmisten, überblicken und aktuell halten

Anwender sollten die Anzahl der Apps so gering wie möglich halten. So behalten sie einen guten Überblick über ihren gesamten App-Fuhrpark. Zudem werden weniger persönliche Daten über die installierten Apps an große Firmen, Spam-versendende Werbeagenturen und potenzielle Angreifer und Betrüger gesendet. Je weniger Apps auf dem Mobilgerät installiert sind, desto geringer das Risiko. Darüber hinaus sollten alle genutzten Apps immer aktualisiert werden, um mögliche Programmlücken abzudichten.

Fünfte Sicherheitsregel: Drittan bieter-Stores vermeiden Nutzer sollten Apps ausschließlich aus den offiziellen App-Stores beziehen. Vor allem Android-Nutzer sollten die Funktion „Installation von Apps von anderen Quellen als Play Store erlauben“ nur in Ausnahmesituationen aktivieren.

Sechste Sicherheitsregel: Apps professionell prüfen Android-Nutzer sollten zusätzliche Schutzvorkehrungen über mobile Virenschutzprogramme wie z.B. Kaspersky Internet Security for Android treffen. Damit wird jede neu heruntergeladene App genauestens geprüft. Zudem sollten Anwender immer ganz genau die Berechtigungen prüfen, die jede App fordert. Somit wird vermieden, dass Apps mehr Informationen verlangen als sie benötigen.

\section{Deutscher Mittelstand fordert betrieblichen Datenschutzbeauftragten auf EU-Ebene}

Der Berufsverband der Datenschutzbeauftragten Deutschlands e.V. (BvD) sieht sich in seiner Forderung, die Funktion des Datenschutzbeauftragten europaweit umfassend zu verankern, durch den JAHRESMITTELSTANDSBERICHT 2014 bestätigt.

In dem am06.06.2014 von der Arbeitsgemeinschaft Mittelstand - eine Gruppierung aus zehn Verbänden des produzierenden und dienstleistenden Gewerbes - veröffentlichten Bericht werden u.a. im Entwurf der EU-Kommission einer Datenschutzgrundverordnung zum Datenschutzbeauftragten Verbesserungen angemahnt. Die Arbeitsgemeinschaft Mittelstand sieht es als problematisch an, wenn durch die geplante EU-Datenschutzgrundverordnung Melde-, Beratungs- und Kontrollaufgaben nur noch über Aufsichtsbehörden und nicht durch einen selbst gewählten, betrieblichen Datenschutzbeauftragten erfüllt werden können. 